\title{
Effect of spacer layer thickness on the static characteristics of resonant tunneling diodes
}

I. Mehdi, R. K. Mains, and G. I. Haddad

Center for High-Frequency Microelectronics, Department of Electrical Engineering and Computer Science, The University of Michigan, Ann Arbor, Michigan 48109

(Received 8 December 1989; accepted for publication 19 June 1990)

\begin{abstract}
A self-consistent quantum mechanical simulation is used to study the effect of spacer layer thickness on such resonant tunneling diode properties as the peak current and peak-to-valley current ratio. It is found that with a low cathode doping the peak current is insensitive to the commoniy used spacer layer thickness. However, for higher cathode doping the peak current decreases with increasing spacer layer thickness. This phenomenon is explained on the basis of the junction potential between the heavily doped cathode contact region and the undoped double-barrier region. Thus, for device applications where a high current density is desired the cathode spacer layer should be designed as thin as possible.
\end{abstract}

Various two and three terminal device applications have been proposed and demonstrated which utilize the current-voltage $I(V)$ characteristic of the double-barrier resonant tunneling structure (DBRTS). ${ }^{1,2}$ For most of these appiications the static characteristic of the DBRTS remains important. For instance the peak current and the negative resistance are important in determining the $f_{\max }$ and stability of the resonant tunneling diode oscillator. ${ }^{3}$ Similarly the peak current of the structure limits the maximum collector current available from the resonant tunneling transistor (RTT) and the valley current results in the leakage current where the transistor is in the off state. ${ }^{4}$ The purpose of this letter is to report on a systematic study of the effect of spacer layer thickness $L_{s}$ on the $I(B)$ characteristic of the device using a self-consistent quantum mechanical simulation. Spacer layers were originally used to decrease the diffusion of dopant impurities into the doublebarrier undoped region. Spacer layers will also, by virtue of increasing the depletion width, decrease the diode capacitance making the device more suitable for high-frequency operation. Long spacer layers are also found to be useful in the fabrication of RTTs. Muto et al. ${ }^{5}$ experimentally studied the effect of varying the spacer layer thickness on one side of a GaAs/AiGaAs device at $77 \mathrm{~K}$ and found that the peak current decreased with increasing spacer layer thickness when the spacer was on the cathode side. However, no explanation was provided for this behavior. It was also reported that the peak-to-valley current ratio increased with increasing spacer layer thickness.

The numerical simulation that is used has been described in detall by Mains and co-workers. ${ }^{6.7}$ The overall numerical procedure is briefy described here. An initial guess is generated for the potential and electron concentrations in the contacts, and the quantum well concentration is initially assumed to be zero. The Newton-Raphson iterative method is used to solve the set of nonlinear equations describing the conduction band and the electron concentration in the contacts. The new electron concentration is used to calculate the conduction band which in turn then determines the electron concentration. This process is re- peated until the maximum fractional change of potential and carrier density predicted by the Newton-Raphson method for each iteration is below a specified tolerance. After convergence is obtained, the device current density is calculated by solving the time-independent Schrödinger equation using the finite difference method described in Ref. 6.

The structure investigated corresponds to a GaAs/ AlGaAs device. The barriers are selected to be $26.1 \AA$ wide and $0.24 \mathrm{eV}$ high. The well is $49.3 \AA$ wide. The electron mass is taken to be $0.092 m_{0}$ in the barriers and $0.067 m_{0}$ elsewhere. The spacer layer thickness is varied on both sides of the doubie-barrier structure.

The peak current density $J_{p}$ as a function of $L_{s}$ with the doping in the cathode (and anode) as a parameter is shown in Fig. 1. At low doping levels $J_{p}$ does not change as a function of $L_{s}$. However, as the doping is increased $J_{p}$ becomes more sensitive to the variation in the spacer iayer thickness. Figure 2(a) shows the conduction-band profile

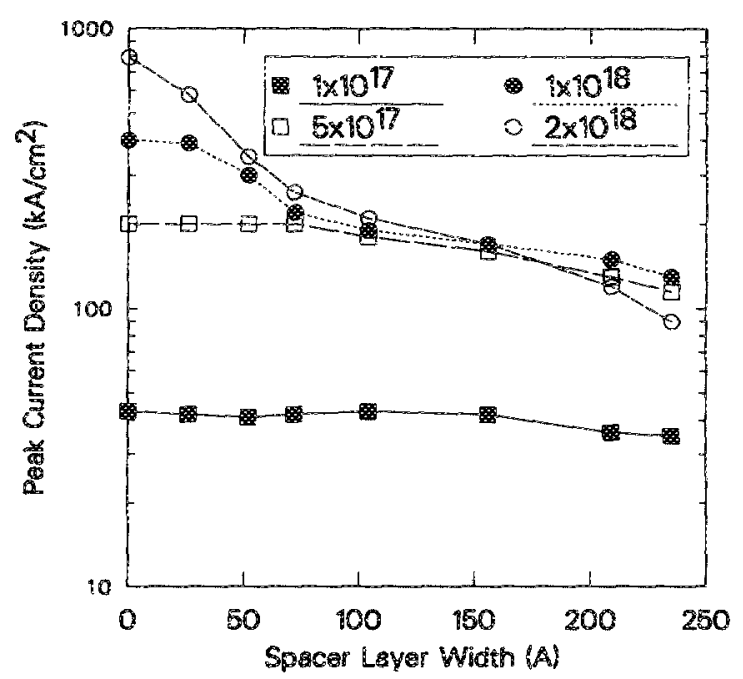

FIG. 1. Effect of spacer layer width on the peak current density of resonant tunneling diodes. 


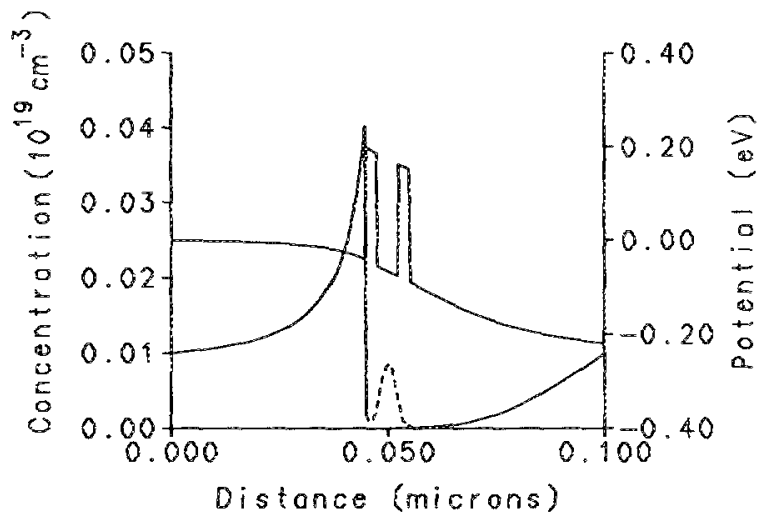

(a)

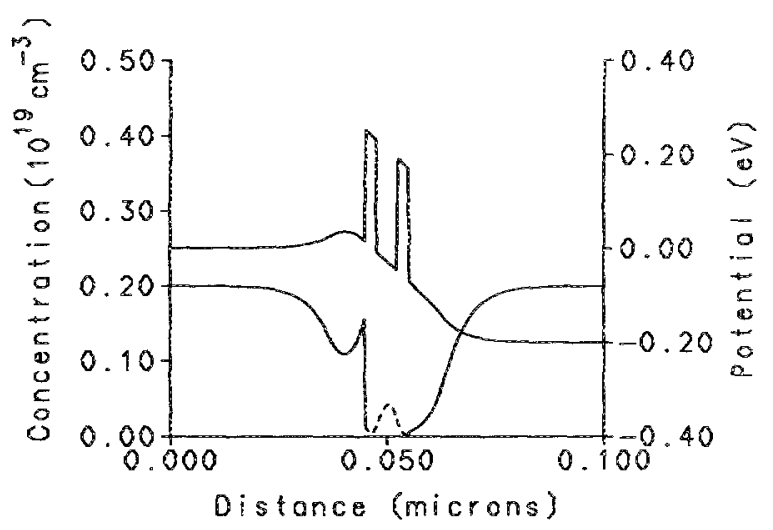

(b)

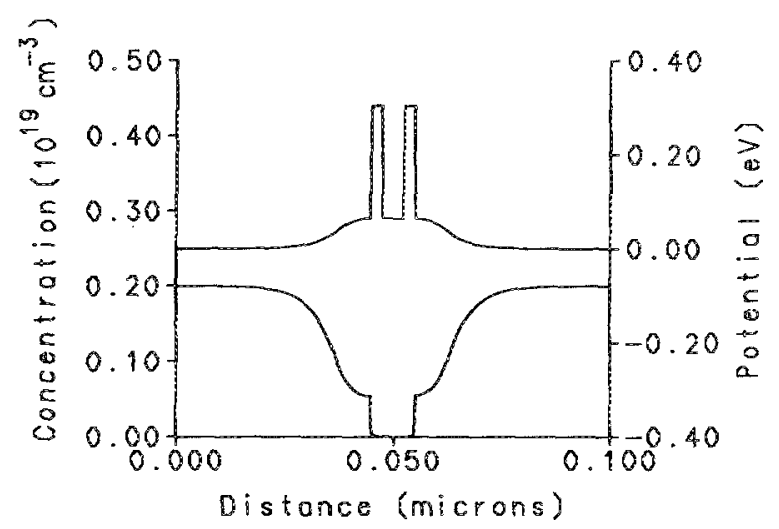

(c)

FIG. 2. Conduction-band diagram for the structure under study (a) $78 \AA$ spacer layers, doping is $1 \times 10^{17} \mathrm{~cm}^{3}$, biased at resonance, (b) $78 \AA$ spacer layers, doping is $2 \times 10^{18} \mathrm{~cm}^{-3}$, biased at resonance, (c) $78 \AA$ spacer layers, doping is $2 \times 10^{18} \mathrm{~cm}^{3}$, zero applied bias.

and electron concentration of the DBRTS biased at resonance with a doping of $1 \times 10^{17} \mathrm{~cm}^{-3}$ for a case with a spacer layer of $78 \AA$ on both sides. In this case, like the structure with no spacer layers (not shown) an accumulation region is formed at the cathode. In the same structure biased at resonance with no spacer layers where now the cathode doping is increased to $2 \times 10^{18} \mathrm{~cm}^{-3}$ the accumulation region is still found to exist. However, when in this structure of high doping, spacer layers of $78 \AA$ are introduced the accumulation region in the cathode actually becomes a depletion region as shown in Fig. 2(b). The elec- trons tunneling from the cathode now see an added barrier and thus the current density is decreased. The conduction band profile for a heavily doped sample $\left(2 \times 10^{18} \mathrm{~cm}^{-3}\right)$ at zero bias is shown in Fig. $2(\mathrm{c})$. Due to the contact potential at the $n^{+}-i$ junction the double-barrier region is situated on a "hill-like structure." At resonance part of the potential drop is across the $n^{+}-i$ junction. For heavily doped devices this potential is not enough to completely overcome the junction potential, causing a decrease in the current density. Consequently, for device applications requiring a high peak current density the contact doping should be made large and the cathode spacer layer should be made as thin as possible.

The effect of spacer layer variation on the peak-tovalley ratio (PTVR) and depletion region width $D_{\text {dep }}$ of the structure is listed in Table $\mathbb{l}$. The PTVR does not seem to be a strong function of the spacer layer thickness for at least the commonly used thicknesses. The slight variation in the calculated ratios is an attribute of the numerical procedure. However, it should be noted that the simulation does not take into account any scattering processes and thus experimentally it might be possible to observe an increase in the PTVR ratio with increasing spacer layer thickness. This would be possible because the spacer layer will decrease the diffusion of dopant impurities into the double-barrier structure which will result in a reduction of the ionized impurity scattering. The depletion region edge on both sides is calculated to be the point where the carrier concentration falls to half the value of the concentration in the contact regions. The depletion width, as expected, is a strong function of both the doping and the spacer layer thickness.

For comparison purposes devices with asymmetric spacer layers have been studied. In these devices cathode spacer layer can be made as thin as possible and the anode spacer layer thickness can be increased. Figure 3 shows the

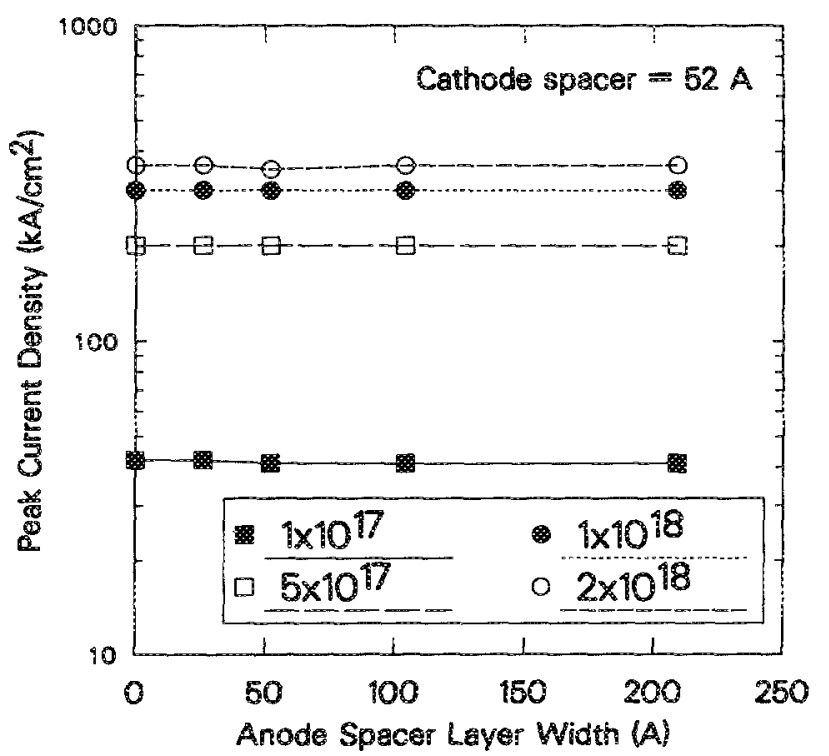

FIG. 3. Effect of anode spacer layer width on the peak current density. The cathode spacer layer was fixed at $52 \AA$. 
TABLE I. Effect of spacer layer width on the PTVR and depietion region width ( $D_{\text {dep }}$ ) of a RTD for various doping concentrations. The depletion regionis given in Angstroms.

\begin{tabular}{|c|c|c|c|c|c|c|c|c|}
\hline \multirow{3}{*}{$\begin{array}{c}\text { Spacer } \\
\text { widih } \\
(\mathrm{A})\end{array}$} & \multicolumn{8}{|c|}{ Cathode and anode doping } \\
\hline & $1 \times 10^{17}$ & $\mathrm{~cm}^{-3}$ & $5 \times 10^{17}$ & $\mathrm{~cm}^{-3}$ & $1 \times 10^{18}$ & $\mathrm{~cm}^{-3}$ & $2 \times 10^{18}$ & $\mathrm{~cm}^{-3}$ \\
\hline & PTVR & $D_{\text {dep }}$ & PTVR & $D_{\text {dep }}$ & PTVR & $D_{\text {dep }}$ & PTVR & $D_{\text {iep }}$ \\
\hline 0 & 9.1 & 470 & 8.0 & 250 & 7.6 & 214 & 6.4 & 194 \\
\hline 26 & 9.0 & 498 & 8.1 & 271 & 7.5 & 230 & 4.8 & 204 \\
\hline 52 & 8.9 & 527 & 8.2 & $30:$ & 5.2 & 253 & 4.1 & 219 \\
\hline 78 & 9.1 & 543 & 8.2 & 329 & 4.9 & $27 !$ & 4.2 & 236 \\
\hline 104 & 9.4 & 568 & 6.3 & 351 & 4.5 & 296 & 4.6 & 256 \\
\hline 156 & 9.7 & 605 & 5.9 & 400 & 5.3 & 345 & 5.7 & 306 \\
\hline 209 & 9.2 & 628 & 5.6 & 452 & 5.6 & 396 & 5.5 & 356 \\
\hline 235 & 9.0 & 639 & 5.5 & 476 & 5.5 & 420 & 4.6 & 384 \\
\hline
\end{tabular}

calculated peak current density for a device with a fixed cathode spacer layer of $52 \AA$ as a function of the anode spacer layer thickness. Notice that now the peak current is a function of the device doping and is unaffected by the anode spacer layer. A drawback of this design is that the device is not symmetrical anymore which is desirabie for certain applications. The PTVR of such devices was found to be fairly constant as a function of anode spacer layer thickness. This contrasts with the $77 \mathrm{~K}$ experimental results presented in $\mathbb{R e}$. 5 . However, one mast remember that the simulation does take into account any scattering effects and thus experimentally it is possible to observe increasing PTVR with increasing spacer layer thickness as discussed above.

In summary, we have presented a systematic study of the effect of spacer layer thickness variation on the static $I(V)$ characteristic of the RTD. For low cathode doping the peak current is not sensitive to the spacer layer thickness. However, for higher cathode doping due to the junction potential between the $n^{+}-i$ layers the peak current becomes sensitive to the spacer layer thickness. This information can be very useful for designing high-frequency devices that utilize the $I(V)$ characteristic of the doublebarrier resonant tunneling structure.

This work was supported by the U.S. Army Research Offee under the URI program, contract No. DAAL03-87. $\mathrm{K}-0007$.

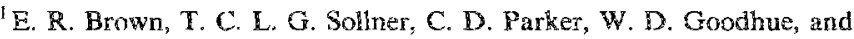
C. L. Chen, Appl. Phys. Lett. 55, 1777 (1989).

${ }^{2}$ F. Capasso, S. Sen, F. Beltram, L. M. Lunardi, A. S. Vengurlekar, P. R. Smith, N. J. Shah, R. J. Malik, and A. Y. Cho, IEEE Trans. Electron Devices, 36, 2065 (1989).

${ }^{3} \mathrm{C}$. Kidner, I. Mehdi, J. East, and G. Haddad, IEEE Trans. Microwave Theory Tech. 38, 854 (1990).

${ }^{4}$ G. I. Haddad, R. K. Mains, U. K. Reddy, and J. R. Easis, J. Superlatt. Microstruct. 5, 437 (1989).

${ }^{5}$ S. Muto, T. Inata, H. Ohnisni, N. Yokoyama, and S. Hiyamizu, Jpn. I. Appl. Phys. 25, 1577 (1986).

${ }^{6}$ R. K. Mains and G. I. Haddad, J. Appl. Phys. 64, 3564 (1988).

${ }^{7}$ R. K. Mains, J. P. Sun, and G. I. Haddad, Appl. Phys. Lett. 55, 371 (1989). 